\title{
RANG RÉEL DE CERTAINES EXTENSIONS
}

\author{
NAWFAL ELHAGE HASSAN
}

(Communicated by Palle E. T. Jorgensen)

\begin{abstract}
RÉsumÉ. On montre pour toutes $C^{*}$-algebras $A$ et $J$ l'égalité entre les rangs réels de $A$ et de $A / J$ si $J$ est essentiel dans $A$ et isomorphe à l'algèbre des opérateurs compacts. En particulier, le rang réel de l'algèbre de Toeplitz $\mathscr{T}$ est égal à 1 .
\end{abstract}

\section{INTRODUCTION ET NOTATION}

L. G. Brown et G. K. Pedersen [1] ont introduit le rang réel que l'on note (rr), comme une notion non commutative de dimension pour les $C^{*}$-algèbre. En fait, ils ont montré que le rang réel d'une $C^{*}$-algèbre commutative et unitaire est égal à la dimension de recouvrement de son spectre, affirmant ainsi le principe selon lequel une $C^{*}$-algèbre est "un espace localement compact non commutatif". Ils ont aussi montré que si $A$ est une $C^{*}$-algèbre et $J$ est un idéal bilatère fermé dans $A$, alors $\operatorname{rr}(A)=0$ si et seulement $\operatorname{si} \operatorname{rr}(J)=\operatorname{rr}(A / J)=0$ et si tout projecteur dans $A / J$ se relève par un projecteur dans $A$. On va traiter essentiellement dans cette note le comportement du rang réel par rapport à certaines suites exactes. On démontre en 1.4 que pour toute $C^{*}$-algèbra $A$ et tout idéal bilatère fermé $J$ dans $A$, on a $\operatorname{Max}\{\operatorname{rr}(J), \operatorname{rr}(A / J)\} \leq \operatorname{rr}(A)$. On prouve en 1.7 que, si $A$ est commutative, alors on a $\operatorname{rr}(A)=\operatorname{Max}\{\operatorname{rr}(J), \operatorname{rr}(A / J)\}$, ceci équivaut au fait que, si $X$ est un espace compact et $U$ est un ouvert dans $X$, alors on a $\operatorname{dim}(X)=\operatorname{Max}\left\{\operatorname{dim}\left(U^{+}\right), \operatorname{dim}(X \backslash U)\right\}$. Ensuite, on démontre en 1.12 que, si $J$ est essentiel dans $A$ et isomorphe à $\mathscr{K}(\mathscr{H})$, alors on a $\operatorname{rr}(A)=\operatorname{rr}(A / J)$. En particulier, on a $\operatorname{rr}(\mathscr{T})=1$, où $\mathscr{T}$ est l'algèbre de Toeplitz.

Soient $A, J$ deux $C^{*}$-algèbres, on note $A^{+}$la $C^{*}$-algèbre obtenue par adjonction d'une unité à $A, \widetilde{A}=A^{+}$si $A$ n'est pas unitaire et $\widetilde{A}=A$ si $A$ est unitaire, et $A_{s a}$ (resp. $A_{+}$) l'ensemble des éléments hermitiens (resp. positifs ou nuls) dans $A . J<A$, signifie que $J$ est un idéal bilatère fermé dans la $C^{*}$-algèbre $A$. On dit que $J$ est un idéal essentiel dans $A$ si $J<A$ et si tout idéal bilatère fermé non nul dans $A$ a une intersection non nulle avec $J$ ([6], 3.12.7). $\mathscr{M}(A)$ la $C^{*}$-algèbre des multiplicateurs de $A$ et $A^{\prime \prime}$ l'algèbre de von Neumann enveloppante de $A$, alors on a $\mathscr{M}(A) \subset A^{\prime \prime}$ [6]. Si $A$ est unitaire, on note $\mathrm{GL}(A)$ l'ensemble des éléments inversibles dans $A, \mathrm{GL}(A)_{+}=\mathrm{GL}(A) \cap A_{+}$

Received by the editors November 1, 1993 and, in revised form, March 17, 1994.

1991 Mathematics Subject Classification. Primary 46L05. 
et $L g_{n}(A)$ l'ensemble des $n$-uples d'éléments de $A$ qui engendrent $A$ en tant qu' idéal à gauche et on pose $g h_{n}(A)=L g_{n}(A) \cap\left(A_{s a}\right)^{n}$.

$$
\text { c.à.d., } g h_{n}(A)=\left\{\left(x_{1}, x_{2}, \ldots, x_{n}\right) \in\left(A_{s a}\right)^{n} / \sum_{i=1}^{n} x_{i}^{2} \in \mathrm{GL}(A)_{+}\right\} \text {. }
$$

On note $\mathscr{K}(\mathscr{H})$ (resp. $\mathscr{L}(\mathscr{H})$ ) la $C^{*}$-algèbre des opérateurs compacts (resp. continus) sur l'espace hilbertien $\mathscr{H}$, et si $\mathscr{H}$ est séparable de diension infinie, on pose $\mathscr{K}(\mathscr{H})=\mathscr{K}$.

Définition [1]. Soit $A$ une $C^{*}$-algèbre unitaire. Le rang réel de $A$, noté $\operatorname{rr}(A)$, est le plus petit entier $n \geq 0$ (s'il existe) tel que $g h_{n+1}(A)$ soit dense dans $\left(A_{s a}\right)^{n+1}$. S'il n'existe aucun $n \geq 0$ tel que $g h_{n+1}(A)$ soit dense dans $\left(A_{s a}\right)^{n+1}$, on pose $\operatorname{rr}(A)=+\propto$. Si $A$ est non unitaire, on pose $\operatorname{rr}(A)=\operatorname{rr}(\widetilde{A})$.

Remarque. Pour toute $C^{*}$-algèbre $A$, on a $\operatorname{rr}(A)=\operatorname{rr}\left(A^{+}\right)$.

Définition [5]. Soit $X$ un espace topologique non vide, la dimension de recouvrement de $X$, notée $\operatorname{dim}(X)$, est le plus petit entier $n \geq 0$ (s'il existe) tel que l'on ait la propriété suivante:

$\left(\mathbf{P}_{n}\right)$ : pour tout recouvrement ouvert $U_{1}, \ldots, U_{k}$ de $X$, il existe un recouvrement ouvert $V_{1}, \ldots, V_{k}$ de $X$ tel que $V_{i} \subset U_{i}$ pour tout $i$ et pour tout $x \in X$, il existe au plus $(n+1)$ éléments de $\left\{V_{1}, \ldots, V_{k}\right\}$ contenant $x$. S'il n'existe aucun $n \geq 0$ tel que l'on ait $\left(\mathrm{P}_{n}\right)$, on pose $\operatorname{dim}(X)=+\propto$. Si $X=\phi$, la dimension de $\bar{X}$ est par définition égal à -1 .

\section{RANG REEL DE CERTAINES EXTENSIONS}

1.1. Lemme [4]. Soient $A$ une $C^{*}$-algèbre et $n \geq 0$. Etant donnés $\varepsilon>0$ et $a=\left(1+a_{0}, a_{1}, \ldots, a_{n}\right) \in \overline{g h_{n+1}(\widetilde{A})}$ avec $a_{i} \in A_{s a}$, alors il existe $b=$ $\left(1+b_{0}, b_{1}, \ldots, b_{n}\right) \in g h_{n+1}(\widetilde{A})$ avec $b_{i} \in A_{s a}$ tel que $\left\|b_{i}-a_{i}\right\|<\varepsilon$ pour $i=0, \ldots, n$.

1.2. Lemme [4]. Soient $A$ une $C^{*}$-algèbre, $n \geq 0$ et $1=1_{A^{+}}$. Alors les conditions suivantes sont équivalentes:

(i) $\operatorname{rr}(A) \leq n$;

(ii) $\left(1+A_{s a}\right) \times\left(A_{s a}\right)^{n} \subset \overline{g h_{n+1}\left(A^{+}\right)}$;

(iii) $\forall \varepsilon>0, \forall \xi \in\left(1+A_{s a}\right) \times\left(A_{s a}\right)^{n}$, il existe $\eta=\left(1+a_{0}, a_{1}, \ldots, a_{n}\right) \in$ $g h_{n+1}\left(A^{+}\right)$avec $a_{i} \in A_{s a}(i \in\{0,1, \ldots, n\})$ tel que $\|\xi-\eta\|<\varepsilon$.

1.3. Proposition. Soient $A$ une $C^{*}$-algèbre, $J<A$ et $n \geq 0$, alors on a:

$\operatorname{rr}(J) \leq n \quad$ si et seulement si $\left(1+J_{s a}\right) \times\left(J_{s a}\right)^{n} \subset \overline{g h_{n+1}(\widetilde{A})}$.

Démonstration. La démonstration est inspirée de ([7], 4.4). La condition est clairement nécessaire, montrons sa suffisance. On peut supposer $1 \in A$ (car $J<A^{+}$) et donc $\tilde{A}=A$. On peut aussi supposer $J \neq A$, car sinon, c'est clair.

Soient $d=\left(1+d_{0}, d_{1}, \ldots, d_{n}\right) \in\left(1+J_{s a}\right) \times\left(J_{s a}\right)^{n}$ et $\varepsilon>0$. D'après 1.2 , il suffit de démontrer qu'il existe $a \in\left[g h_{n+1}\left(J^{+}\right)\right] \cap\left[\left(1+J_{s a}\right) \times\left(J_{s a}\right)^{n}\right]$ tel que $\|d-a\|<\varepsilon$. Par hypothèse, il existe $\left(1+a_{0}, a_{1}, \ldots, a_{n}\right) \in g h_{n+1}(A)$ tel que $\left\|d_{i}-a_{i}\right\|<\varepsilon / 2$, pour $i=0,1, \ldots, n$.

Soit $\left(u_{\lambda}\right)_{\lambda \in \Lambda}$, une unité approchée pour $J([6], 1.4)$, alors il existe $\lambda_{0} \in$ $\Lambda$ tel que pour tout $\lambda \geq \lambda_{0}$, on ait $\left\|u_{\lambda} d_{i}-d_{i}\right\|<\varepsilon / 2$ et $\left\|u_{\lambda} d_{i} u_{\lambda}-d_{i}\right\|<$ 
$\varepsilon / 2 \quad(i=0, \ldots, n)$. Puisque $\left(1+a_{0}, a_{1}, \ldots, a_{n}\right) \in g h_{n+1}(A)$, alors il existe $b_{0}, b_{1}, \ldots, b_{n}$ dans $A$ tels que

$$
\left(1+b_{0}\right)\left(1+a_{0}\right)+b_{1} a_{1}+\cdots+b_{n} a_{n}=1 \text {, d'où on a } b_{0}+a_{0}+\sum_{i=0}^{n} b_{i} a_{i}=0 .
$$

Pour tout $i \in\{0, \ldots, n\}$, on a $u_{\lambda 0} b_{i} \in J$, donc il existe $\lambda_{1} \in \Lambda$ tel que pour tout $\lambda \geq \lambda_{1}$, on ait $\left\|u_{\lambda 0} b_{i}-u_{\lambda 0} b_{i} u_{\lambda}\right\|<\varepsilon / 2$ pour tout $i \in\{0, \ldots, n\}$. Désormais, on travaille avec $\lambda \in \Lambda$ tel que $\lambda \geq \lambda_{0}, \lambda_{1}$. On a donc $\| u_{\lambda} a_{i} u_{\lambda}-$ $u_{\lambda} d_{i} u_{\lambda}\|\leq\| a_{i}-d_{i} \|<\varepsilon / 2$, d'où on a $\left\|d_{i}-u_{\lambda} a_{i} u_{\lambda}\right\|<\varepsilon$ pour tous $i \in\{0, \ldots, n\}$ et $\lambda \geq \lambda_{0}, \lambda_{1}$. Montrons qu'alors

$$
a_{\lambda}=\left(1+u_{\lambda} a_{0} u_{\lambda}, u_{\lambda} a_{1} u_{\lambda}, \ldots, u_{\lambda} a_{n} u_{\lambda}\right)
$$

est dans $g h_{n+1}\left(J^{+}\right)$pour tout $\lambda \geq \lambda_{0}, \lambda_{1}$.

On a $u_{\lambda 0} b_{0} u_{\lambda}+u_{\lambda 0} a_{0} u_{\lambda}+\sum_{i=0}^{n} u_{\lambda 0} b_{i} a_{i} u_{\lambda}=0$. D'où on a:

$$
\begin{aligned}
\| 1- & {\left[\left(u_{\lambda 0} b_{o}+1\right)\left(1+u_{\lambda} a_{0} u_{\lambda}\right)+\sum_{i=1}^{n} u_{\lambda 0} b_{i} u_{\lambda} a_{i} u_{\lambda}\right] \| } \\
= & \left\|u_{\lambda 0} b_{0}+u_{\lambda} a_{0} u_{\lambda}+\sum_{i=0}^{n} u_{\lambda 0} b_{i} u_{\lambda} a_{i} u_{\lambda}\right\| \\
= & \left\|u_{\lambda 0} b_{0}+u_{\lambda} a_{0} u_{\lambda}+\sum_{i=0}^{n} u_{\lambda 0} b_{i} a_{i} u_{\lambda}-\sum_{i=0}^{n} u_{\lambda 0} b_{i} a_{i} u_{\lambda}+\sum_{i=0}^{n} u_{\lambda 0} b_{i} u_{\lambda} a_{i} u_{\lambda}\right\| \\
= & \left\|u_{\lambda 0} b_{0}+u_{\lambda} a_{0} u_{\lambda}-u_{\lambda 0} b_{0} u_{\lambda}-u_{\lambda 0} a_{0} u_{\lambda}+\sum_{i=0}^{n}\left(u_{\lambda 0} b_{i} u_{\lambda} a_{i} u_{\lambda}-u_{\lambda 0} b_{i} a_{i} u_{\lambda}\right)\right\| \\
\leq & \left\|u_{\lambda 0} b_{0}-u_{\lambda 0} b_{0} u_{\lambda}+u_{\lambda} a_{0} u_{\lambda}-u_{\lambda 0} a_{0} u_{\lambda}\right\| \\
& +\left\|\sum_{i=0}^{n}\left(u_{\lambda 0} b_{i} u_{\lambda} a_{i} u_{\lambda}-u_{\lambda 0} b_{i} a_{i} u_{\lambda}\right)\right\| .
\end{aligned}
$$

Posons:

$$
x=\left\|u_{\lambda 0} b_{0}-u_{\lambda 0} b_{0} u_{\lambda}+u_{\lambda} a_{0} u_{\lambda}-u_{\lambda 0} a_{0} u_{\lambda}\right\|
$$

et

$$
y=\left\|\sum_{i=0}^{n}\left(u_{\lambda 0} b_{i} u_{\lambda} a_{i} u_{\lambda}-u_{\lambda 0} b_{i} a_{i} u_{\lambda}\right)\right\|,
$$

alors on a:

$$
\begin{aligned}
x & \leq\left\|u_{\lambda 0} b_{0}-u_{\lambda 0} b_{0} u_{\lambda}\right\|+\left\|u_{\lambda} a_{0} u_{\lambda}-u_{\lambda 0} a_{0} u_{\lambda}\right\| \\
& \leq\left\|u_{\lambda 0} b_{0}-u_{\lambda 0} b_{0} u_{\lambda}\right\|+\left\|u_{\lambda} a_{0}-d_{0}\right\|+\left\|d_{0}-u_{\lambda 0} a_{0}\right\| .
\end{aligned}
$$

On a $\left\|u_{\lambda} a_{0}-u_{\lambda} d_{0}\right\| \leq\left\|a_{0}-d_{0}\right\|<\varepsilon / 2 \quad \forall \lambda \in \Lambda$ et $\left\|u_{\lambda} d_{0}-d_{0}\right\|<\varepsilon / 2 \quad \forall \lambda \geq \lambda_{0}$.

D'où pour tout $\lambda \geq \lambda_{0}$, on a $\left\|u_{\lambda} a_{0}-d_{0}\right\|<\varepsilon$. D'où on a $x \leq 3 \varepsilon / 2$.

On a:

$$
\begin{aligned}
y & \leq \sum_{i=0}^{n}\left\|u_{\lambda 0} b_{i} u_{\lambda} a_{i} u_{\lambda}-u_{\lambda 0} b_{i} a_{i} u_{\lambda}\right\| \leq \sum_{i=0}^{n}\left\|u_{\lambda 0} b_{i} u_{\lambda} a_{i}-u_{\lambda 0} b_{i} a_{i}\right\| \\
& \leq \sum_{i=0}^{n}\left\|u_{\lambda 0} b_{i} u_{\lambda}-u_{\lambda 0} b_{i}\right\|\left\|a_{i}\right\| .
\end{aligned}
$$


Soit $\delta=\operatorname{Max}\left\{\left\|d_{i}\right\| ; i=0, \ldots, n\right\}$, alors on a $y \leq(n+1)(\varepsilon / 2)[\delta+(\varepsilon / 2)]$. D'où pour tout $\lambda \geq \lambda_{0}, \lambda_{1}$, on $\mathbf{a}$ :

$\left\|1-\left[\left(u_{\lambda 0} b_{0}+1\right)\left(1+u_{\lambda} a_{0} u_{\lambda}\right)+\sum_{i+1}^{n} u_{\lambda 0} b_{i} u_{\lambda} a_{i} u_{\lambda}\right]\right\|<(\varepsilon / 2)[(n+1)(\delta+(\varepsilon / 2))+3]$.

Donc pour $\varepsilon>0$ assez petit tel que $(\varepsilon / 2)[(n+1)(\delta+(\varepsilon / 2))+3]<1$, on a:

$$
a_{\lambda} \in\left[g h_{n+1}\left(J^{+}\right)\right] \cap\left[\left(1+J_{s a}\right) \times\left(J_{s a}\right)^{n}\right] \text { et }\left\|d-a_{\lambda}\right\|<\varepsilon \text {. }
$$

1.4. Théorème. Soient $A$ une $C^{*}$-algèbre et $J<A$, alors on a:

$$
\operatorname{Max}\{\operatorname{rr}(J), \operatorname{rr}(A / J)\} \leq \operatorname{rr}(A) .
$$

Démonstration. $\operatorname{rr}(J) \leq \operatorname{rr}(A)$ résulte immédiatement de la proposition précédente. Supposons d'abord que $A$ est unitaire, alors pour tout $n \geq 1$ on a: $\left((A)_{s a}\right)^{n} \stackrel{\pi}{\rightarrow}\left((A / J)_{s a}\right)^{n}$ est surjectif, continu et $\pi\left(g h_{n}(A)\right) \subset g h_{n}(A / J)$. Donc, si $m=\operatorname{rr}(A)$, on a $g h_{m+1}(A)=\left(.(A)_{s a}\right)^{m+1}$ d'où $g h_{m+1}(A / J)=\left((A / J)_{s a}\right)^{m+1}$, d'où $\operatorname{rr}(A / J) \leq \operatorname{rr}(A)$. Si $1 \notin A$, alors on a $(\tilde{A} / J) \cong(a / J)^{+}$, d'où on a: $\operatorname{rr}(A / J)=\operatorname{rr}\left((a / J)^{+}\right)=\operatorname{rr}(\tilde{A} / J) \leq \operatorname{rr}(\widetilde{A})=\operatorname{rr}(A)$.

Rappelons le résultat suivant dû à [5].

1.5. Proposition. Soient $X$ un espace normal et $Y$ un fermé dans $X$, si $\operatorname{dim}(Y) \leq n$ et si $\operatorname{dim}(F) \leq n$, pour tout fermé $F$ dans $X$ tel que $F \cap Y=\varnothing$, alors on $a \operatorname{dim}(X) \leq n$.

1.6. Corollaire. Soient $X$ un espace compact et $U$ un ouvert dans $X$, alors on $a$ :

$$
\operatorname{dim}(X)=\operatorname{Max}\left\{\operatorname{dim}\left(U^{+}\right), \operatorname{dim}(X \backslash U)\right\} .
$$

Démonstration. On a $\operatorname{Max}\left\{\operatorname{dim}\left(U^{+}\right), \operatorname{dim}(X \backslash U)\right\} \leq \operatorname{dim}(X)$, ceci découle de 1.4, où on prend $A=C(X), J=C_{0}(U)$ et $A / J=C(X \backslash U)$, et du fait que $J^{\sim}=C\left(U^{+}\right)$. Donc il reste à démontrer l'inégalité opposée. On peut supposer $\operatorname{Max}\left\{\operatorname{dim}\left(U^{+}\right), \operatorname{dim}(X \backslash U)\right\}=n$ (fini). On pose $Y=X \backslash U$, donc $Y$ est fermé dans $X$ et on a $\operatorname{dim}(Y) \leq n$. Soit $F$ un fermé dans $X$ tel que $F \cap Y=\varnothing$, alors on a $F \subset U \subset U^{+}, F$ est un espace compact et $U^{+}$est un espace séparé, d'où $F$ est fermé dans $U^{+}$, et alors $\operatorname{dim}(F) \leq \operatorname{dim}\left(U^{+}\right) \leq n$, par 1.4. D'où on a $\operatorname{dim}(X) \leq n$ par 1.5.

Il faut noter qu'il existe un espace compact $X$ et un ouvert $U$ dans $X$ tel que $\operatorname{dim}(U)=1>0=\operatorname{dim}(X)$ ([5], 4.3.1, p. 161).

1.7. Théorème. Soient $A$ une $C^{*}$-algèbre commutative et $J<A$, alors on a:

$$
\operatorname{rr}(A)=\operatorname{Max}\{\operatorname{rr}(J), \operatorname{rr}(A / J)\} .
$$

Démonstration. Si $1 \in A$, ceci découle immédiatement du corollaire précedent. Si $1 \notin A$, alors on a la suite exacte suivante:

$$
0 \rightarrow J \rightarrow A^{+} \rightarrow(A / J)^{+} \rightarrow 0 .
$$

D'où, on a $\operatorname{rr}(A)=\operatorname{rr}\left(A^{+}\right)=\operatorname{Max}\left\{\operatorname{rr}(J), \operatorname{rr}\left((A / J)^{+}\right)\right\}=\operatorname{Max}\{\operatorname{rr}(J), \operatorname{rr}(A / J)\}$.

1.8. Remarques ([6], 3.12). (i) Soient $A$ une $C^{*}$-algèbre et $x \in \mathscr{M}(A)$ tel que $A x=\{0\}$, alors on a $x=0$. Puisque $A x=\{0\}$, on a $\forall a \in A$ ax $=0$. 
D'où, par continuté, $\forall b \in A^{\prime \prime}$, on a $b x=0$. Comme $x \in \mathscr{M}(A) \subset A^{\prime \prime}$, d'où $x^{*} x=0$, donc $x=0$.

(ii) Soient $A$ une $C^{*}$-algèbre et $J<A$, alors on a:

$$
J \subset A \subset \mathscr{M}(J) \Leftrightarrow J \text { est un idéal essentiel dans } A \text {. }
$$

Démonstration. Si $J$ est un idéal essentiel dans $A$, alors on a $A \subset \mathscr{M}(J)$ $\operatorname{par}([6], 3.12 .8)$. Démontrons l'autre sens. Soit $I<A$ tel que $J \cap I=\{0\}$. Soit $x \in I$, alors on a $J x \subset(J \cap I)=\{0\}$, d'où $x=0$ car $x \in A \subset \mathscr{M}(J)$. Donc on a $I=\{0\}$, donc $J$ est un idéal essentiel dans $A$.

(iii) On a $\mathscr{M}(\mathscr{K}(\mathscr{H}))=\mathscr{L}(\mathscr{H})([6], 3.12 .3)$.

(iv) Soient $A$ une $C^{*}$-algèbre et $J<A$ tel que $J \cong \mathscr{K}(\mathscr{H})$, alors par (ii) et (iii) on a:

$$
J \text { est essentiel dans } A \Leftrightarrow \mathscr{K}(\mathscr{H}) \subset A \subset \mathscr{L}(\mathscr{H}) .
$$

Soient $A$ une $C^{*}$-algèbre et $J$ un idéal bilatère fermé dans $A$, de sort que l'on a la suite exacte courte suivante:

$$
0 \rightarrow J \rightarrow A \stackrel{\pi}{\rightarrow} A / J \rightarrow 0 \text {. }
$$

On dit que (1) est triviale (resp. scindée) si $A \cong J \oplus(A / J)$ (resp. s'il existe $\phi \in \operatorname{Hom}(A / J, A)$ tel que $\left.\pi_{0} \phi=\mathrm{id}_{A / J}\right)$.

1.9. Proposition. On suppose que, dans (1), A/J est simple et $J$ est idéal non trivial dans $A$, alors on a:

(i) $J$ n'est pas essentiel dans $A$ si et seulement si (1) est triviale.

(ii) Si $A$ est unitaire et $J$ est non unitaire, alors $J$ est essentiel dans $A$.

Si de plus $J$ est simple, alors $J$ est l'unique idéal bilatère fermé non trivial dans A.

Démonstration. (i) $\mathrm{Si}$ (1) est triviale, alors il est clair que $J$ n'est pas essential dans $A$. Réciproquement, si $J$ n'est pas essentiel dans $A$, alors il existe $I$, idéal bilatère fermé et non nul dans $A$ tel que $I \cap J=\{0\}$. On a $\pi(I)<A / J$ et $\pi(1) \neq\{0\}$, car $I \neq\{0\}$ et $I \cap J=\{0\}$. Pisque $A / J$ est simple, on a $\pi(I)=A / J$. Puisue $\pi_{\mid I}$ est injectif $(I \cap J=\{0\}), \pi_{\mid I}$ est un isomorphisme de $C^{*}$-algèbres de $I$ sur $A / J$, d'où $I \cong A / J$ et $A=I+J$. Comme $I J=$ $I \cap J=\{0\}$ et $I, J<A$, on a $A \cong I \oplus J$. D'où $A \cong J \oplus(A / J)$ et $\pi$ s'identifie avec la projection canonique sur la deuxième composante.

(ii) On suppose maitenant $A$ unitaire et $J$ non unitaire. $\mathrm{Si} J$ n'est pas essentiel, alors d'après (i), on peut supposer que $A=J \oplus(A / J)$.

Puisque $A$ est unitaire ceci entraîne que $J$ est unitaire, ce qui est contraire à l'hypothèse. Donc $J$ est essentiel dans $A$. On suppose de plus que $J$ est simple, et soit $I$ un idéal bilatère fermé non nul dans $A$ tel que $I \neq J$. On a $(I \cap J)<J$, et puisque $J$ est essentiel dans $A$, on a $I \cap J \neq\{0\}$. Puisque $J$ est simple, on a $I \cap J=J$ et alors $J \subset I$. On a $\pi(I)<A / J$ et $\pi(I) \neq\{0\}$ car $I \neq\{0\}$ et $J \neq I$. D'òu $\pi(I)=A / J$, car $A / J$ est simple, et alors $A=I+J=I$ (car $J \subset I)$.

1.10. Remarque. Soit $A$ une $C^{*}$-algèbre, alors on a:

$A$ est essentiel dans $A^{+} \Leftrightarrow A$ est non unitaire.

Ceci résulte immédiatement du (ii) de la proposition précédente. Remarquons que si $A$ est non unitaire la suite exacte $0 \rightarrow A \rightarrow \widetilde{A} \rightarrow \mathbb{C} \rightarrow 0$ est scindée non 
triviale, $\mathbb{C}$ est simple et $A$ est essentiel dans $\tilde{A}$ où $\mathbb{C}$ est le corps de nombres complexes.

1.11. Corollaire. Soient $A$ une $C^{*}$-algèbre unitaire et $J<A$, non unitaire et non nul. Si $A / J$ est simple, alors on a $J \subset A \subset \mathscr{M}(J)$.

Ceci résulte immédiatement de la proposition précédente et de 1.8 (ii).

1.12. Théorème. On suppose que, dans (1), $J$ est un idéal essentiel dans $A$ et isomorphe à $\mathscr{K}(\mathscr{H})$. Alors on a $\operatorname{rr}(A)=\operatorname{rr}(A / J)$.

Démonstration. Grâce à la Remarque 1.8 (iv), on peut supposer $J=\mathscr{K}(\mathscr{H})<$ $A \subset \mathscr{L}(\mathscr{H}) . \mathrm{Si} \operatorname{dim}(\mathscr{H})=n<+\propto$, on a $A=M_{n}(\mathbb{C})$ et $A / J=\{0\}$. D'où on a $\operatorname{rr}(A / J)=\operatorname{rr}(0)=\operatorname{rr}\left(0^{+}\right)=\operatorname{rr}(\mathbb{C})=0=\operatorname{rr}\left(M_{n}(\mathbb{C})\right)=\operatorname{rr}(A)$ ([1], 2.10). Donc on peut supposer que $\operatorname{dim}(\mathscr{H})=+\propto$. Supposons d'abord que $A$ est unitaire (nécessairement $1_{A}=1_{\mathscr{E}}$ car $\mathscr{K}(\mathscr{H})<A$ ). On sait, par le Théorème 1.4, que l'on a $\operatorname{rr}(A / J) \leq \operatorname{rr}(A)$, donc il reste à démontrer l'autre inégalité. On peut supposer $\operatorname{rr}(A / J)=n<+\propto$, parce que sinon on a $\operatorname{rr}(A)=\operatorname{rr}(A / J)=+\propto$.

Soient $\left(h_{1}, h_{2}, \ldots, h_{n+1}\right) \in\left(A_{s a}\right)^{n+1}$ et $\varepsilon>0$. Posons pour $i=1,2, \ldots$, $n+1, B_{i}=\left\{x \in A_{s a} /\left\|h_{i}-x\right\|<\varepsilon\right\}$ et $U=\prod_{i=1}^{n+1} B_{i}$. Puisque $\pi:\left(A_{s a}\right)^{n+1} \rightarrow$ $\left((A / J)_{s a}\right)^{n+1}$ est linéaire, continue et surjective, donc $\pi(U)$ est un ouvert non vide dans $\left((A / J)_{s a}\right)^{n+1}$. Puisque l'on a $n=\operatorname{rr}(A / J)$, il existe

$$
\left(f_{1}, f_{2}, \ldots, f_{n+1}\right) \in(U) \cap g h_{n+1}(A / J) .
$$

Donc il existe $x_{i} \in B_{i}$ tel que $f_{i}=\pi\left(x_{i}\right)$ pour $i=1,2, \ldots, n+1$. Posons $x=\sum_{i=1}^{n+1} x_{i}^{2} \in A_{+}$. Puisque $\left(f_{1}, f_{2}, \ldots, f_{n+1}\right) \in g h_{n+1}(A / J)$ et $\pi\left(x_{i}\right)=f_{i}$, on a $\pi(x)=\sum_{i=1}^{n+1} f_{i}^{2} \in \mathrm{GL}(A / J)_{+}$, donc $x$ est opérateur de Fredholm positif. Soit $p=1-q$ où $q$ est le support de $x$, donc on a $p \in J$ et $\left[x+\left((n+1) \varepsilon^{2}\right) p\right] \in$ $\mathrm{GL}(A)_{+}$. Pour $i=1, \ldots, n+1$, on a $x \geq\left(x_{i}\right)^{2} \geq 0$, ceci implique $\left(x_{i}\right) p=0$. Comme $p, x_{i} \in A_{s a}$, ceci entrîne que $p x_{i}=0$.

Pour $i=1, \ldots, n+1$, posons $z_{i}=x_{i}+\varepsilon p \in A_{s a}$, alors on a $\sum_{i=1}^{n+1} z_{i}^{2}=$ $x+\left((n+1) \varepsilon^{2}\right) p \in \mathrm{GL}(A)_{+}$. Donc on a $\left(z_{1}, z_{2}, \ldots, z_{n+1}\right) \in g h_{n+1}(A)$ et $\left\|z_{i}-h_{i}\right\| \leq\left\|z_{i}-x_{i}\right\|+\left\|x_{i}-h_{i}\right\|<2 \varepsilon$. D'où on a $\overline{g h_{n+1}(A)}=\left(A_{s a}\right)^{n+1}$. Donc on a $\operatorname{rr}(A) \leq n=\operatorname{rr}(A / J)$, d'où $\operatorname{rr}(A)=\operatorname{rr}(A / J)$. Si $1 \notin A$, on a $(\widetilde{A}) / J \cong(A / J)^{+}$, et alors on a $\operatorname{rr}(A)=\operatorname{rr}(\widetilde{A})=\operatorname{rr}((\widetilde{A}) / J)=\operatorname{rr}\left((A / J)^{+}\right)=\operatorname{rr}(A / J)$.

1.13. Corollaire. On a les propriétés suivantes:

(i) si $A$ est lalgèbre de Toeplitz $\mathscr{T}$, on a $\operatorname{rr}(A)=1$,

(ii) si $A$ est lune des algèbres $O_{n}$ de Cuntz [3] $(n \geq 2)$, on a $\operatorname{rr}(A)=$ $\operatorname{rr}\left(O_{n}\right)=0=\operatorname{rr}\left(O_{\propto}\right)$.

Démonstration. (i) On a la suite exacte bien connue.

$$
0 \rightarrow \mathscr{K} \rightarrow \mathscr{T} \rightarrow C(T) \rightarrow 0 .
$$

Avec $\mathscr{T} \subset \mathscr{M}(\mathscr{K})$, d'où on a $\operatorname{rr}(\mathscr{T})=\operatorname{rr}(C(T))=\operatorname{dim}(T)=1$.

(ii) On a la suite exacte suivante:

$$
0 \rightarrow \mathscr{K} \rightarrow A \rightarrow O_{n} \rightarrow 0([2], 3.1) .
$$

$O_{n}$ est une $C^{*}$-algèbre simple, unitaire et purement infinie ([2], 1.13). D'où on a $\operatorname{rr}\left(O_{n}\right)=0([1], 3.9)$. De même on a $\operatorname{rr}\left(O_{\propto}\right)=0$. Puisque $O_{n}$ est simple et 
$\mathscr{K}$ non unitaire, alors d'après 1.9(ii), $\mathscr{K}$ est un idéal essentiel dans $A$, d'où par 1.12, on a $\operatorname{rr}(A)=\operatorname{rr}(A / \mathscr{K})=\operatorname{rr}\left(O_{n}\right)=0$.

1.14. Corollaire. Soient $\mathscr{H}$ un espace hilbertien séparable de dimension infinie et $n \in \mathscr{N} \cup\{\propto\}$, alors il existe une sous- $C^{*}$-algèbre unitaire $A$ dans $\mathscr{L}(\mathscr{H})$ telle que $\mathscr{K}<A \subset \mathscr{L}(\mathscr{H}), \operatorname{rr}(A)=n$ et $A /(\mathscr{K})$ est commutative.

Démonstration. Soit $X$ un espace compact et métrqiue de dimension $n$ (par exemple $\left.X=[0,1]^{n}\right)$, alors $X$ est séparable et il existe une suite $\left(x_{n}\right)_{n \in \mathscr{N}}$ dense dans $X$ telle que chaque $x_{n}$ est répété une infinité de fois. Soient $\mathscr{H}=l^{2}(\mathscr{N}), \pi: \mathscr{L}(\mathscr{H}) \rightarrow \mathscr{L}(\mathscr{H}) / \mathscr{K}$, la surjection canonique et $\Lambda: C(X) \rightarrow$ $\mathscr{L}(\mathscr{L}) / \mathscr{K}$, définie par: $\Lambda(f)=\pi\left(\operatorname{diag}\left(f\left(x_{n}\right)\right)\right)$. Alors $\Lambda$ est un morphisme de $C^{*}$-algèbres. Soit $f \in C(X)$ tel que $\Lambda(f)=0$, alors $\operatorname{diag}\left(f\left(x_{n}\right)\right)$ est un opérateur compact et comme chaque $x_{n}$ est répété une infinité de fois, on a $f=0$, d'où $\Lambda$ est injective. Posons $A=\pi^{-1}(\Lambda(C(X)))$, alors $A$ est une $C^{*}$-algèbre unitaire dans $\mathscr{L}(\mathscr{H})$ contenant $\mathscr{K}$ et on a la suite exacte suivante:

$$
0 \rightarrow \mathscr{K} \rightarrow A \rightarrow C(X) \rightarrow 0 .
$$

D'où, d'après le Théorème 1.12, on a $\operatorname{rr}(A)=\operatorname{rr}(C(X))=\operatorname{dim}(X)=n$.

Face à tous ces résultats, on ne sait pas répondre à la question suivante: Existe-t-il une $C^{*}$-algèbre $A$ et $J$ un idéal bilatère fermé dans $A$ tel que l'on ait

$$
\operatorname{rr}(A)>\operatorname{Max}\{\operatorname{rr}(J), \operatorname{rr}(A / J)\} ?
$$

\section{ACKNOWLEDGMENT}

Ce travail fait partie de ma thèse [4], préparée sous la direction du Professeur Georges Zeller-Meier que je remercie vivement.

\section{REFERENCES}

1. L. G. Brown et G. K. Pedersen, $C^{*}$-algebras of real rank zero, J. Funct. Anal. 99 (1991), 131-149.

2. J. Cuntz, Simple $C^{*}$-algebras generated by isometries, Comm. Math. Phys. 57 (1977), 173-185.

3. _ K- K-theory for certain $C^{*}$-algebras, Ann. of Math. (2) 113 (1981), 181-197.

4. N. Elhage Hassan, Rangs stables des $C^{*}$-algèbres, Thèse, Université d'Aix-Marseille-II, 1993.

5. A. R. Pears, Dimension theory of general spaces, Cambridge Univ. Press, Cambridge, 1975.

6. G. K. Pedersen, $C^{*}$-algebras and their automorphism groups, Academic Press, London, 1979.

7. M. A. Rieffel, Dimension and stable rank in the $K$-theory of $C^{*}$-algebras, Proc. London. Math. Soc. (3) 46 (1983), 301-333.

Département de Mathématiques, Université d’Orléans, B.P. 6759,45067 Orléans Cedex 2, France

E-mail address: Nawfal@Labomath.univ-orleans.fr 